\title{
Aa. Vv., «George Sand Studies», Vol. 28
}

\section{Morena Petrich}

\section{(2) OpenEdition}

\section{Journals}

\section{Edizione digitale}

URL: http://journals.openedition.org/studifrancesi/6041

DOI: 10.4000/studifrancesi.6041

ISSN: 2421-5856

\section{Editore}

Rosenberg \& Sellier

\section{Edizione cartacea}

Data di pubblicazione: 1 mai 2011

Paginazione: 196-197

ISSN: 0039-2944

\section{Notizia bibliografica digitale}

Morena Petrich, «Aa. Vv., «George Sand Studies», Vol. 28», Studi Francesi [Online], 163 (LV | I) | 2011

online dal 30 mai 2015, consultato il 09 janvier 2021. URL: http://journals.openedition.org/

studifrancesi/6041 ; DOI: https://doi.org/10.4000/studifrancesi.6041

Questo documento è stato generato automaticamente il 9 janvier 2021.

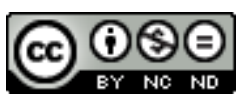

Studi Francesi è distribuita con Licenza Creative Commons Attribuzione - Non commerciale - Non opere derivate 4.0 Internazionale. 


\title{
Aa. Vv., «George Sand Studies», Vol. 28
}

\author{
Morena Petrich
}

\section{NOTIZIA}

AA. VV., «George Sand Studies», Vol. 28, 2009, pp. 94.

Il nuovo numero della rivista «George Sand Stu-dies» si apre con un interessante studio di Kate BoNIN (The Edifying Spectacle of a Drowned Woman: Sympathy and Irony in "Indiana", pp. 1-13). Nato come vibrante denuncia dello statuto subalterno imposto alle donne dalla legislazione del tempo, attraverso il vincolo del matrimonio, Indiana riprende il topos dell'isola lontana, già messo in scena da Rousseau con l'Émile e da Bernardin de Saint-Pierre con Paul et Virginie, il cui ricordo riecheggia nelle ultime parole della protagonista sandiana. L'A. insiste però sulla funzione dello strumento ironico nel romanzo, che rimanda a molteplici e suggestive interpretazioni e che, intrecciandosi col sapiente ricorso di Sand al doppio e al simbolo, incarna un preciso e costante monito a rifuggire dalla presunta univocità delle parole. Anche il secondo studio che incontriamo (Writing Back: Female Self-Authorship in "Paul et Virginie" and "Indiana", pp. 14-26) mette in relazione le stesse due opere, Indiana e Paul et Virginie: Anne MARColine vi invita a scorgere nel romanzo sandiano un'amplificazione del testo di Bernardin, una sorta di riscrittura dal punto di vista della protagonista, un passaggio di testimone tra $\mathrm{i}$ due personaggi femminili e la progressione di un percorso di consapevolezza. Emerge così la rivendicazione autoriale, che si profila come un preciso attacco all'intera struttura patriarcale dell'epoca: la scrittura a partire dal sé femminile assume la fisionomia di un atto eminentemente politico e rivoluzionario, volto a scuotere l'intero impianto concettuale e simbolico dell'Ancien Régime. Véronique MACHELIDON propone una ricca analisi del concetto di alterità ancora nel romanzo d'esordio di Sand (George Sand's Praise of Creoleness: Race, Slavery and (In)Visibility in "Indiana", pp. 27-45), incentrata su una rivisitazione delle categorie di razza, schiavitù, 
inferiorità. Adottando un approccio al contempo psicoanalitico, decostruzionista e storicistico, l'A. getta nuova luce sull'impegno di Sand nella lotta alle discriminazioni razziali e sociali. L'insistenza della scrittrice sui concetti di ibrido, "créolité" e doppio ne svela il sapiente uso per contrastare e smontare ogni fondamento somatico o etnico della diversità, denunciando al contempo le pesanti implicazioni sessiste e discriminatorie dell'imperante simbologia patriarcale e degli interessi colonialisti. In Nouvelles lectures du sphinx: George Sand et Henry James (pp. 46-56) Maryline LUKACHER sottolinea come l'immagine della sfinge ritorni sia nei romanzi di Sand (in particolare ne La Famille de Germandre) che nelle descrizioni che i suoi contemporanei fecero della personalità e della bellezza della loro autrice. Lukacher stabilisce un parallelo tra Sand e Henry James, ammiratore di "Old George", insistendo principalmente sulle affinità riscontrate tra le opere dei due letterati ed esplicitando il valore della sfinge nei loro scritti, non solo in quanto emblema di enigmi e suggestioni magiche, ma anche come veicolo di seduzione e angoscia.

2 Il tema del denaro è l'asse portante di Nanon, romanzo del 1872, nelle cui pagine emerge con forza il rapporto di Sand con le questioni di ordine finanziario: su tale aspetto investiga con il suo contributo ("Nanon" (1872): une défense du capitalisme? Réflexions sur la signification de l'argent dans l'œuvre ainsi qu'autour de l'œuvre, pp. 57-64) di Manon MATHIAS. Così come per la protagonista della storia, l'indipendenza economica è sempre stata una priorità anche per l'autrice: il suo profondo "realismo economico" si riflette negli innumerevoli riferimenti alle somme di denaro guadagnate dalla giovane nel corso della sua ascesa economica e sociale. Per la scrittrice, così come per il personaggio letterario, il guadagno è sia una legittima necessità che uno strumento per la soddisfazione dei proprio bisogni e per la realizzazione dei proprio sogni; il professionismo, difeso ed assunto come valore, non viene mai ad intaccare né l'autonomia dell'artista né la profonda valenza artistica delle sue opere. Si realizza così, nella piena maturità della romanziera, quell "'idealismo pragmatico ed economico" che si affianca all'altro, più noto e a giusto titolo celebrato, politico-sociale del suo messianico engagement. Sempre in contesto economico-politico si inquadra lo studio di Marie-Claire valloIs ("Fanchette": Critique de l'économie politique (1839-1850), pp. 65-76), che verte sul romanzo "villageois" pubblicato per la prima volta nel 1843. Come ricorda Vallois, nella prefazione al testo Sand ricorre a termini che rimandano alla sfera religiosa e alla parola biblica, che affianca ad altri prettamente "secolari". L'avvicendarsi delle sfere semantiche e dei relativi ambiti di riferimento funge da amplificatore del messaggio paritetico ed egualitarista sandiano, che, avvalendosi del linguaggio evangelico per propagare il verbo repubblicano, mette polemicamente in scena la "parole prolétaire". In chiusura del numero, Xavier vEzzoli interroga le fonti letterarie e gli scritti epistolari per chiarire l'infondata e ancor oggi assai diffusa condanna di Sand per la morte prematura di Chopin (George Sand et la mort de Chopin, selon Liszt, pp. 77-83). L'A. evidenzia come la voce sull'influsso nefasto ed il peso determinante del loro addio sulla salute, in realtà già da tempo pesantemente compromessa, del musicista sia scaturita per la prima volta dalla biografia che -Liszt scrisse nel 1852, tre anni dopo il decesso di Chopin.

Nelle pagine del testo, Liszt indugia sulla disperazione del compositore polacco per la rottura con Sand del 1847, dolore dal quale non si sarebbe più ripreso; l'attenta $\mathrm{e}$ dettagliata disamina proposta da Vezzoli dimostra invece che ciò non influì in maniera preponderante sulle delicate condizioni di Chopin. Lo studio evidenzia anche come l'enfasi posta dall'improvvisato biografo sul mal d'amore in relazione alla scomparsa 
del musicista polacco vada piuttosto rivalutata in considerazione di ragioni di ordine editoriale, in primis l'interesse di incontrare il gusto del pubblico, dei lettori e soprattutto delle lettrici.

3 La rivista si chiude infine con la presentazione di tre recenti pubblicazioni, rispettivamente di Isabelle HOOG NAGINSKI (George Sand mythographe, Clermont-Ferrand, Presses Universitaires Blaise Pascal, 2007, pp. 273), di Christine PLANTÉ (George Sand critique 1833-1876. Textes de George Sand sur la littérature présentés, édités et annotés sous la direction de Christine Planté, Tusson, Charente, Du Lérot éditeur, 2006, pp. 804), Catherine NESCI (Le Flâneur et les flâneuses: Les femmes et la ville à l'époque romantique, Grenoble, ELLUG, 2007, pp. 400). 